\title{
Decoupling of superfluid and normal modes in pulsating neutron stars
}

\author{
Mikhail E. Gusakov ${ }^{1}$ and Elena M. Kantor ${ }^{1,2}$ \\ ${ }^{1}$ Ioffe Physical Technical Institute, Polytekhnicheskaya 26, 194021 St.-Petersburg, Russia \\ ${ }^{2}$ St.-Petersburg State Polytechnical University, Polytekhnicheskaya 29, 195251 St.-Petersburg, Russia
}

\begin{abstract}
We show that equations governing pulsations of superfluid neutron stars (NSs) can be split into two sets of weakly coupled equations, one describing the superfluid modes and another one, the normal modes. The coupling parameter $s$ is small, $|s| \sim 0.01-0.05$, for realistic equations of state (EOSs). Already an approximation $s=0$ is sufficient to calculate the pulsation spectrum within the accuracy of a few percent. Our results indicate, in particular, that emission of gravitational waves from superfluid pulsation modes is suppressed in comparison to that from normal modes. The proposed approach allows to drastically simplify modeling of pulsations of superfluid NSs.
\end{abstract}

PACS numbers: 97.60.Jd, 47.75.+f, 97.10.Sj, 47.37.+q

Introduction. - The pulsations of NSs can be excited either due to internal instabilities or owing to external perturbations. Currently the detectors that will be able, according to preliminary estimates, to register gravitational waves from pulsating NSs, are under development [1]. For the correct interpretation of future observations it is necessary to have a well developed theory of NS pulsations. The formulation of such a theory is complicated by the fact that at a temperature $T \lesssim 10^{8}-10^{10} \mathrm{~K}$, baryons in the internal layers of NSs become superfluid. Thus, to model pulsations one has to employ superfluid hydrodynamics which is much more complicated than the ordinary one, describing "normal" (nonsuperfluid) matter.

For the first time the global pulsations of superfluid NSs were analyzed by Lindblom and Mendell in 1994 [2]. Considering a simple model of a Newtonian star they numerically found two distinct classes of pulsation modes: (i) normal modes which practically coincide with the corresponding modes of a normal star; and, (ii) superfluid modes in which the matter pulsates in such a way that the mass current density approximately vanishes. The subsequent numerical studies of various pulsation modes (the literature is vast; see, e.g., Refs. [3, 4] and references therein) confirmed the result of Ref. [2] though general explanation of this result has not yet been proposed [5]. In this work we give such an explanation. In addition, we present an approximate scheme which allows to greatly simplify calculations of pulsating superfluid NSs. In what follows the speed of light $c=1$.

Superfluid hydrodynamics. - For simplicity, we consider NS cores composed of neutrons $(n)$, protons $(p)$, and electrons $(e)$. We also assume that protons are normal while neutrons are superfluid in some region of a NS core. As demonstrated in Ref. [6] possible admixture of other particle species (e.g., muons) and proton superfluidity do not affect our principal results. Finally, we first consider a nonrotating NS. Effects of rotation will be incorporated later in the text.

It is well known that in superfluid matter, several independent motions with different velocities may coexist without dissipation [7]. In our case the system is fully described by two four-vectors, $u^{\mu}$ and $w_{(n)}^{\mu}$. The vector $u^{\mu}$ is the velocity of electrons and protons as well as "normal" neutrons; the vector $w_{(n)}^{\mu}$ arises from additional degrees of freedom associated with neutron superfluidity. In the nonrelativistic limit the spatial components of the four-vector $w_{(n)}^{\mu}$ are related to superfluid velocities $V_{s n}$ of the Landau-Khalatnikov theory [7] by the equality $\boldsymbol{w}_{(n)}=m_{n}\left(\boldsymbol{V}_{s n}-\boldsymbol{u}\right)$, where $m_{n}$ is the neutron mass; $\boldsymbol{u}$ is the spatial component of the "normal" fourvelocity $u^{\mu}$. The electron $j_{(e)}^{\mu}$, proton $j_{(p)}^{\mu}$, and neutron $j_{(n)}^{\mu}$ current densities are expressed through the vectors $u^{\mu}$ and $w_{(n)}^{\mu}$ as [8, 9] : $j_{(e)}^{\mu}=n_{e} u^{\mu}, j_{(p)}^{\mu}=n_{p} u^{\mu}$, and $j_{(n)}^{\mu}=n_{n} u^{\mu}+Y_{n n} w_{(n)}^{\mu}$. Here $n_{l}$ is the number density of particles $l=n, p$, or $e$. The expression for $j_{(n)}^{\mu}$ consists of two terms reflecting the fact that both normal and superfluid liquid components contribute to neutron current density. The coefficient $Y_{n n}$ has been calculated in Ref. [10]; it is a relativistic analogue of superfluid density of neutrons $\rho_{s n}$. In the nonrelativistic limit $Y_{n n}=\rho_{s n} / m_{n}^{2}$. This coefficient depends on $T$ and increases steadily from 0 for normal matter (when $T \geq T_{c n}$, where $T_{c n}$ is the neutron critical temperature) to $n_{n} / \mu_{n}$ for entirely superfluid matter $(T=0)$. Here and below, $\mu_{l}$ is the chemical potential for particles $l=n, p$, or $e$.

To proceed further, we assume that: (i) the quasineutrality condition holds both for equilibrium and pulsating matter, $n_{p}=n_{e}$; and, (ii) an unperturbed NS is in beta-equilibrium, i.e. the disbalance of chemical potentials $\delta \mu \equiv \mu_{n}-\mu_{p}-\mu_{e}=0$. It is convenient then to formulate the system of nondissipative hydrodynamic equations using the baryon number density $n_{b} \equiv n_{n}+n_{p}$ and $\delta \mu$ as independent variables [9]. The system consists of (1) the continuity equations for baryons and electrons

$$
j_{(b) ; \mu}^{\mu}=0, \quad j_{(e) ; \mu}^{\mu}=0,
$$

where the baryon current density is $j_{(b)}^{\mu} \equiv j_{(n)}^{\mu}+j_{(p)}^{\mu}=$ $n_{b} u^{\mu}+Y_{n n} w_{(n)}^{\mu} ;(2)$ Einstein equations

$$
R^{\mu \nu}-\frac{1}{2} g^{\mu \nu} R=-8 \pi G T^{\mu \nu}
$$


with the energy-momentum tensor $T^{\mu \nu}=(P+\varepsilon) u^{\mu} u^{\nu}+$ $P g^{\mu \nu}+Y_{n n}\left(w_{(n)}^{\mu} w_{(n)}^{\nu}+\mu_{n} w_{(n)}^{\mu} u^{\nu}+\mu_{n} w_{(n)}^{\nu} u^{\mu}\right) ;$ (3) the potentiality condition for superfluid motion of neutrons

$$
\partial_{\nu}\left[w_{(n) \mu}+\mu_{n} u_{\mu}\right]-\partial_{\mu}\left[w_{(n) \nu}+\mu_{n} u_{\nu}\right]=0,
$$

and (4) the second law of thermodynamics

$$
d \varepsilon=\mu_{n} d n_{b}-\delta \mu d n_{e}+\underline{\underline{T d S}}+\underline{Y_{n n} d\left(w_{(n)}^{\alpha} w_{(n) \alpha}\right) / 2 .}
$$

In Eqs. (2)-(4), $R^{\mu \nu}, R$, and $g^{\mu \nu}$ are Ricci tensor, scalar curvature, and metric tensor, respectively; $\partial_{\mu} \equiv \partial / \partial x^{\mu}$; $G$ is the gravitation constant; $P \equiv-\varepsilon+\mu_{n} n_{b}-\delta \mu n_{e}+$ $\underline{T S}$ is the pressure; $\varepsilon$ and $S$ are the energy and entropy densities, respectively. All the thermodynamic quantities are defined in the comoving frame in which $u^{\mu}=(1,0,0,0)$. This imposes an additional constraint on $w_{(n)}^{\mu}[9], u_{\mu} w_{(n)}^{\mu}=0$. The solution to Eqs. (1)-(4) in the superfluid region should be matched with that in the residual (normal) region of a star. Equations, describing pulsations of normal matter can be obtained from the system (11) -(4) if one puts $Y_{n n}=0$ and ignores the condition (3).

Linear Approximation. - In this work we assume that in the unperturbed NS $\boldsymbol{w}_{(n)}=0$, i.e. velocities of normal and superfluid components coincide. For a nonrotating NS this simply means that both components are at rest. Then it follows from the constraint $u_{\mu} w_{(n)}^{\mu}=0$ that $w_{(n)}^{\mu}$ vanishes in equilibrium, $w_{(n)}^{\mu}=0$ (because $u^{0} \neq 0$ ). As a consequence, the terms in Eq. (44) and in the expression for $T^{\mu \nu}$, underlined with one line, are quadratically small. Similarly, the terms which depend on $T$ and underlined twice, are small in the strongly degenerate matter of NSs and can be omitted [9]. Because of the same reasons one can consider, e.g., the quantities $P$ and $\delta \mu$ as functions of only $n_{b}$ and $n_{e}$ (and neglect their dependence on scalars $w_{(n)}^{\mu} w_{(n) \mu}$ and $\left.T\right)$.

Now we make use of the energy-momentum conservation law $T_{: \nu}^{\mu \nu}=0$ which can be derived from Eq. (2). Composing a vanishing combination $T_{; \nu}^{\mu \nu}+u^{\mu} u_{\nu} T_{; \alpha}^{\alpha \nu}$ and subtracting from it Eq. (3) multiplied by $n_{b} u^{\mu}$, one gets, with the help of Eq. (44) and the expression for $P$,

$$
\begin{aligned}
& -n_{e} \partial_{\mu} \delta \mu-u_{\mu} u^{\nu} n_{e} \partial_{\nu} \delta \mu-n_{e} \delta \mu u^{\nu}\left(u_{\mu}\right)_{; \nu} \\
& +\left(g_{\mu \nu}+u_{\mu} u_{\nu}\right) u^{\alpha} X_{; \alpha}^{\nu}+X^{\nu} u_{\mu ; \nu}+X_{\mu} u_{; \nu}^{\nu} \\
& -n_{b} u^{\nu}\left[\partial_{\nu} w_{(n) \mu}-\partial_{\mu} w_{(n) \nu}\right]=0 .
\end{aligned}
$$

Here $X^{\mu} \equiv \mu_{n} Y_{n n} w_{(n)}^{\mu}$. The obtained "superfluid" equation is very attractive because each term in it depends either on $\delta \mu$ or $w_{(n)}^{\mu}$. Both these quantities are small in a slightly perturbed matter (and vanish in equilibrium). This means that in the linear approximation Eq. (5) does not depend explicitly on the perturbations of the metric $g_{\mu \nu}$ and the four-velocity $u^{\mu}$. Thus, one can replace $g_{\mu \nu}$ and $u^{\mu}$ in Eq. (5) by their equilibrium values. For a nonrotating NS the spatial components of Eq. (5) can be rewritten in a remarkably simple form $(j=1,2,3)$

$$
i \omega\left(\mu_{n} Y_{n n}-n_{b}\right) w_{(n) j}=n_{e} \partial_{j}\left(\sqrt{-g_{00}} \delta \mu\right),
$$

where we assumed that $w_{(n)}^{\mu}$ depends on time $t$ as $w_{(n)}^{\mu} \sim$ $\exp (i \omega t)$. In Eq. (6) all the quantities except for $\delta \mu$ and $w_{(n)}^{\mu}$ are taken in equilibrium. Near the equilibrium, the function $\delta \mu\left(n_{b}, n_{e}\right)$ can be expanded in the Taylor series and presented, in the linear approximation, as

$$
\delta \mu=n_{e}\left(\partial \delta \mu / \partial n_{e}\right)\left(z D_{1}+D_{2}\right) .
$$

Here $z \equiv\left[n_{b} \partial \delta \mu / \partial n_{b}\right] /\left[n_{e} \partial \delta \mu / \partial n_{e}\right] ; D_{1} \equiv \delta n_{b} / n_{b} ; D_{2} \equiv$ $\delta n_{e} / n_{e}$. The symbol $\delta$ in front of some quantity denotes a deviation of this quantity from its equilibrium value. The dimensionless functions $D_{1}$ and $D_{2}$ can be found from Eq. (11) and depend on $w_{(n)}^{\mu}, u^{\mu}$, and $g_{\mu \nu}$. Thus, generally, Eq. (6) is not independent and should be solved together with Einstein equations (2). In the linear approximation Eq. (2) can be written in the following symbolic form: $\delta\left(R^{\mu \nu}-1 / 2 g^{\mu \nu} R\right)=-8 \pi G \delta T^{\mu \nu}$. The left-hand side of this equation contains only perturbations of metric. To write out the right-hand side it is convenient to introduce new independent variables, the four-velocity of baryons $U^{\mu} \equiv j_{(b)}^{\mu} / n_{b}=u^{\mu}+Y_{n n} w_{(n)}^{\mu} / n_{b}$ and $W^{\mu} \equiv Y_{n n} w_{(n)}^{\mu} / n_{b}$, instead of $u^{\mu}$ and $w_{(n)}^{\mu}$. Note that for an unperturbed star $U^{\mu}=u^{\mu}$ and $W^{\mu}=0$ (since in equilibrium $w_{(n)}^{\mu}=0$ ). The same is also true for a pulsating NS if the matter is nonsuperfluid (because then $Y_{n n}=0$ ). Employing the new variables an expression for $\delta T^{\mu \nu}$ takes the form

$$
\begin{aligned}
& \delta T^{\mu \nu}=(\delta P+\delta \varepsilon) U^{\mu} U^{\nu} \\
& +(P+\varepsilon)\left(U^{\mu} \delta U^{\nu}+U^{\nu} \delta U^{\mu}\right)+\delta P g^{\mu \nu}+P \delta g^{\mu \nu} .
\end{aligned}
$$

Here the quantities $P, \varepsilon, U^{\mu}$ and $g^{\mu \nu}$ are taken at equilibrium. As follows from Eq. (4) the variation $\delta \varepsilon$ equals $\delta \varepsilon=\mu_{n} \delta n_{b}$ and depends on $\delta U^{\mu}$ and $\delta g^{\mu \nu}$ (and is independent of $\left.\delta W^{\mu}\right)$. The variation $\delta P$ can be expanded in analogy with Eq. (7),

$$
\delta P=n_{b}\left(\partial P / \partial n_{b}\right)\left(D_{1}+s D_{2}\right),
$$

where the function $D_{1}$ depends on $\delta U^{\mu}$ and $\delta g^{\mu \nu}$, and $D_{2}$ depends on the difference $\left(\delta U^{\mu}-\delta W^{\mu}\right)$ and $\delta g^{\mu \nu}$ [see the definitions for $D_{1}$ and $D_{2}$, and Eq. (11)]. The parameter $s$, hereafter referred to as the "coupling parameter", is given by $s \equiv\left(n_{e} \partial P / \partial n_{e}\right) /\left(n_{b} \partial P / \partial n_{b}\right)$.

Superfluid and normal modes. - If $s=0$ then Eq. (8) for $\delta T^{\mu \nu}$ does not depend on $\delta W^{\mu}$ and has exactly the same form as in the absence of superfluidity. In that case the Einstein equations (and boundary conditions to them) coincide, in the linear approximation, with the corresponding equations for normal matter. They can be solved separately from the "superfluid" Eq. (6) so that the solution (the spectrum of eigenfrequencies $\omega$ and the eigenfunctions $\delta U^{\mu}$ ) will be indistinguishable from that for a nonsuperfluid star.

Let us now focus on the following question. Assume that $s$ still vanishes. Is it possible for a NS to oscillate on a frequency which is not an eigenfrequency of a normal star? Suppose that it is indeed the case. Then the linearized Einstein equations will be satisfied only if 


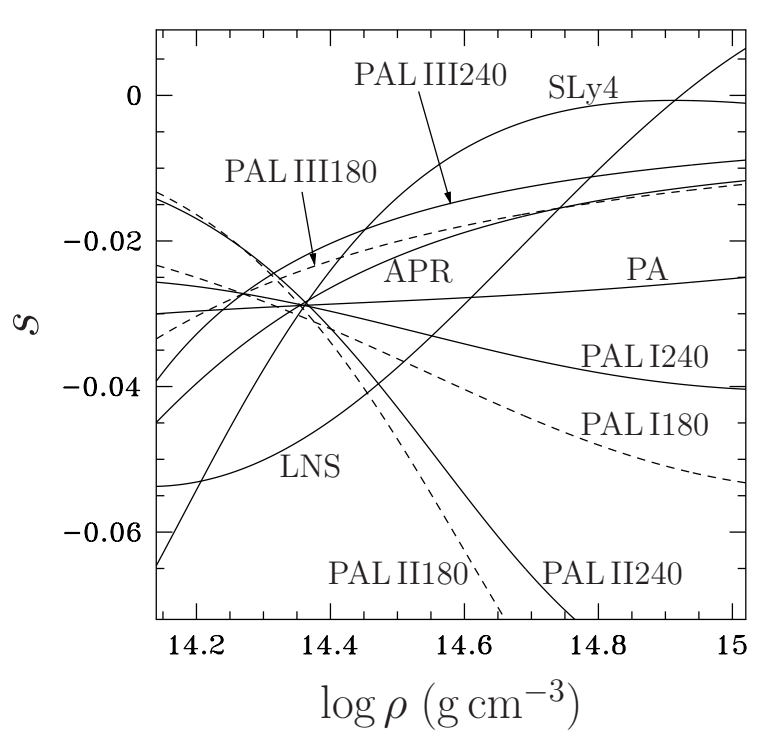

FIG. 1: The coupling parameter $s$ versus density $\rho$ for EOSs PAL 11], PA [12], APR [13], SLy4 [14], and LNS 15]. EOSs of the PAL family differ by the symmetry energy (models I, II, or III) and by the value of the compression modulus, 180 or $240 \mathrm{MeV}$. The models with the compression modulus 120 $\mathrm{MeV}$ are not plotted since they give too small maximum NS masses that seem to contradict observations [16]. Note that the recently measured mass $M=(1.97 \pm 0.04) M_{\odot}$ [17] of the millisecond pulsar PSR J1614-2230 further rules out PA EOS and all PAL EOSs except for PAL I240 and PAL II240.

$\delta U^{\mu}=0$ and $\delta g^{\mu \nu}=0$. As follows from Eq. (1), in this case $\delta n_{b}=0$ (i.e. $D_{1}=0$ ), while $D_{2}$ depends only on $\delta W^{\mu}$ (or, in other words, on $W^{\mu}$, since $W^{\mu}=0$ in equilibrium). In particular, for a nonrotating NS

$$
D_{2}=\left[\left(\partial_{j} n_{e} / n_{e}\right) W^{j}+W_{; j}^{j}\right] /\left(i \omega U^{0}\right) .
$$

Here $j=1,2$, and 3 ; all the quantities, except for $W^{j}$, are taken in equilibrium; when calculating the covariant derivative one should use the unperturbed metric. Eqs. (7) and (10) allow to formulate Eq. (6) purely in terms of $W^{j}$. A boundary condition to this equation, $\boldsymbol{W}_{\perp}=0$, also depends only on $W^{j}$ and can be obtained from the requirement that the baryon current density $j_{(b)}^{\mu}$ is continuous through the normal-superfluid interface $\left(\boldsymbol{W}_{\perp}\right.$ is the component of a vector $W^{j}$, perpendicular to the interface). Thus, Eq. (6) is self-contained and can be solved independently of Eq. (2). Its solution (eigenfrequencies and eigenfunctions $W^{j}$ ) describes superfluid modes which were first considered in Ref. [2] and do not have an analogue for a normal star. To our best knowledge, the striking properties of such modes have not been discussed for a realistic model of a general relativistic NS at finite $T$. First of all, the superfluid pulsation modes do not perturb metric $\left(\delta g^{\mu \nu}=0\right)$ and hence cannot emit gravitational waves. In addition, because for these modes $\delta U^{\mu}=0$ and $\delta n_{b}=0$, the variations of $j_{(b)}^{\mu}$ and $P$ vanish, $\delta j_{(b)}^{\mu}=0$ and $\delta P=0$ [see Eqs. (11) and (9)]. As a consequence, pulsations are localized entirely in the superfluid region of a star. In particular, they do not go to the NS surface.
In the consideration above we supposed that $s=0$. Yet, it is clear that superfluid and normal modes should remain approximately decoupled also at small but finite $s$. As follows from Fig. 1, $s$ is indeed small for realistic EOSs and changes, on the average, from -0.01 to -0.05 [18]. Taking into account that the parameter $z$ in Eq. (7) is $z \sim-1$ for the same EOSs, it is easy to show that for normal modes $D_{1} \gtrsim D_{2}$ [then the second term in Eq. (9]) is much smaller than the first one], while for superfluid modes $D_{1} \sim s D_{2}$ [then the first term in Eq. (7) is much smaller than the second one]. Generally, the exact solution of linearized pulsation equations (22) and (6) can be presented as a series in parameter $s$ [6]. However, since $s$ is very small, the approximation of noninteracting Eqs. (2) and (6) considered above (hereafter "zero approximation") is already sufficient to calculate the pulsation spectrum within the accuracy $\sim s$ (i.e., a few percent).

Example: Radial pulsations. - Let us illustrate the obtained results with an example of a radially pulsating NS with the mass $M=1.4 M_{\odot}$. We consider a simple NS model which was analyzed in detail in Ref. [8]. In that paper it was assumed that the redshifted critical temperature of neutrons $T_{c n}^{\infty}$ is constant throughout the stellar core, $T_{c n}^{\infty}=6 \times 10^{8} \mathrm{~K}$. The results of approximate calculation of pulsation spectrum are illustrated in Fig. 2a. The spectrum is calculated in zero approximation in $s$. In the figure, the pulsation frequency $\omega$ (in units of $\omega_{0} \equiv c / R_{\mathrm{NS}}$, where $R_{\mathrm{NS}}=12.17 \mathrm{~km}$ is the circumferential radius of a star) is plotted as a function of internal redshifted stellar temperature $T^{\infty}$ for 3 normal (solid lines) and 6 superfluid (dashes) pulsation modes. At $T^{\infty}>T_{c n}^{\infty}$ only the normal modes (I, II, and III) survive since then the star is nonsuperfluid. For comparison, in Fig. 2b we present the exact solution to the system of linearized equations (21) and (6). The first 6 modes are shown by alternate solid and dashed lines. The spectrum was not plotted in the shaded region. All other notations are the same as in Fig. 2a. It is easy to see that the structure of both spectra is very similar. However, there is one principal difference. Instead of crossings of superfluid and normal modes in Fig. 2a, we have avoided crossings in Fig. 2b. At these points the superfluid mode becomes normal and vice versa. Such avoided crossings are not described in approximate treatment (Fig. 2a) because when frequencies of superfluid and normal modes are close to each other, Eqs. (2) and (6) become strongly interacting and cannot be considered as independent. For comparison, we plot both spectra in Fig. 2c. The exact solution is shown by solid lines, dashes correspond to the approximate solution. Other notations are the same as in Figs. $2 \mathrm{a}$ and $2 \mathrm{~b}$. On average, the approximate solution differs from the exact one by $\sim 1.5-2 \%$. For normal modes the difference becomes smaller with increasing of $T$. In this case the number of "superfluid" neutrons decreases $\left(Y_{n n} \rightarrow 0\right)$, consequently, $W^{j} \equiv Y_{n n} w_{(n)}^{j} / n_{b} \rightarrow 0$ and zero approximation works better and better.

Taking into account rotation. - Rotation leads to formation of Feynman-Onsager vortices inside NSs with 

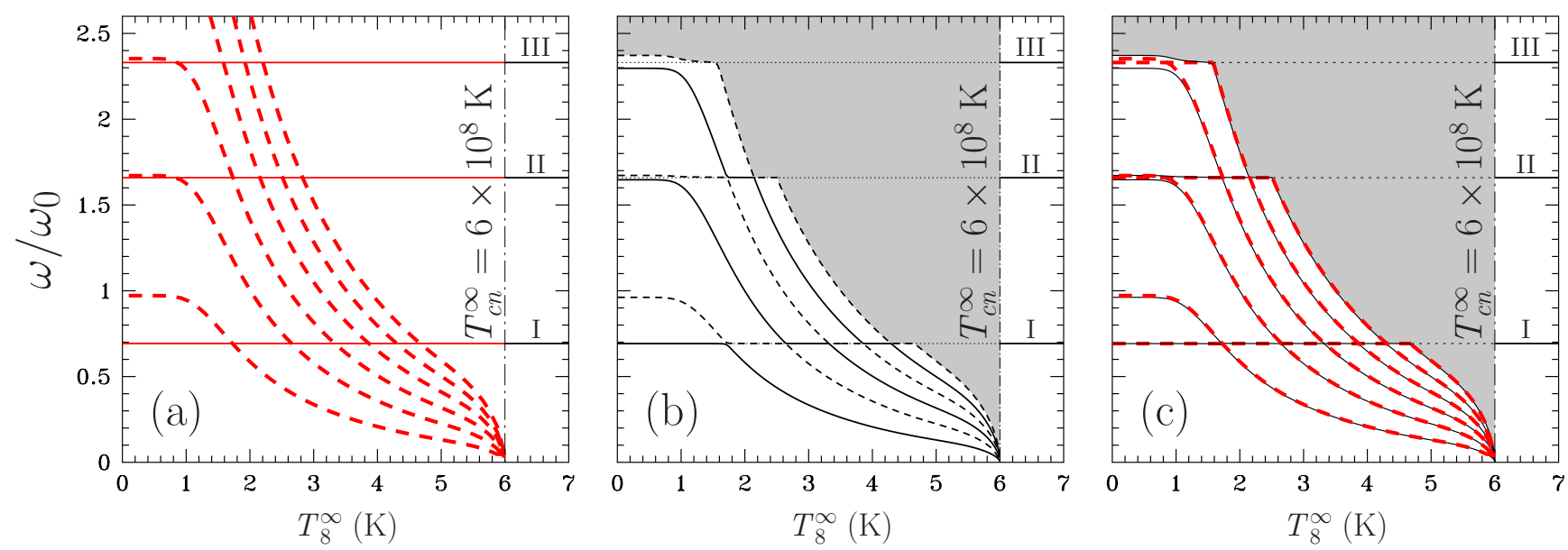

FIG. 2: Frequency $\omega$ in units of $\omega_{0}$ versus $T_{8}^{\infty} \equiv T^{\infty} /\left(10^{8} \mathrm{~K}\right)$ for various pulsation modes. (a) approximate spectrum; (b) exact spectrum; (c) approximate (dashed lines) and exact (solid lines) spectra. For more details see the text.

the interspacing distance $\sim 10^{-2}-10^{-4} \mathrm{~cm}$. The hydrodynamic equations averaged over the volume containing large amount of vortices formally have the same form as in their absence [19] (if we neglect the small contribution of vortices to the internal energy density of matter). The only exception is the potentiality condition (3) that should be replaced by $u^{\nu}\left\{\partial_{\mu}\left[w_{(n) \nu}+\mu_{n} u_{\nu}\right]\right.$ $\left.-\partial_{\nu}\left[w_{(n) \mu}+\mu_{n} u_{\mu}\right]\right\}=O_{\mu \nu} W^{\nu}$, where the tensor $O_{\mu \nu}$ is specified in Ref. [6] and is responsible for the interaction between the normal and superfluid component. It can be found from the requirement that the entropy does not decrease. Because of this condition the new term $n_{b} O_{\mu \nu} W^{\nu}$ appears in the right-hand side of Eq. (5). Since this term depends on a small quantity $W^{\mu}$, all our reasoning about decoupling of superfluid and normal modes remain valid for rotating NSs as well.

Comparison with previous works. - For comparison we choose two papers, Refs. [20] and [4], since at first sight it is not clear whether our results complement or contradict the conclusions drawn in these references.

The authors of Ref. 20] considered a model of Newtonian star at $T=0$. They demonstrated that superfluid modes decouple from the normal modes only for an idealized case of nonstratified NSs, for which $n_{e} / n_{b}=$ const throughout the stellar core.

This result does not contradict ours because one can show that the neutron-star matter is nonstratified only if $\partial P\left(n_{b}, n_{e}\right) / \partial n_{e}=0$ (that is $s=0$ ). As follows from our analysis, in the latter case superfluid and normal modes are indeed strictly decoupled.

The second conclusion made in Ref. [20] is based on the observation that for most of the neutron-star models the stellar matter is stratified. Using this observation the authors of Ref. 20] argued that generally there should be no clear distinction between the superfluid and normal modes, or, in other words, equations describing superfluid- and normal-type pulsations are strongly interacting.

This conclusion is not correct because, as we demonstrated earlier in this work, the real coupling parameter $s$ can be small even for strongly stratified NSs (and is indeed small for realistic EOSs).

Now let us discuss the results of Ref. [4]. This paper analyzed gravitational radiation from superfluid nonrotating NSs at $T=0$ in the frame of the general relativity. It was argued that superfluid modes must radiate gravitational waves in practically all situations, with intensity of radiation comparable to that from the normal modes (unless an EOS has a very specific form satisfying Eq. (74) of Ref. [4]).

When modeling the neutron-star pulsations the authors of Ref. [4] used toy-model EOSs that give completely unrealistic values for the coupling parameter $s$. In particular, we found that their most realistic model II gives $s \sim 0.1$ at the center and $s=\infty$ at the superfluidnormal interface. Moreover, because their EOSs are artificial, they were forced to relax an assumption of chemical equilibrium in the core. As it is demonstrated in the present paper, the latter assumption is very important for the decoupling of modes and cannot be ignored. Thus, it is not surprising that our results disagree with the results of Ref. [4]; when $s$ is not small, superfluid modes can be as effective in radiating gravitational waves as normal modes.

In the end, it is worth mentioning one more result of Ref. [4]. In that paper it is claimed that any (nonradial) pulsation mode must emit gravitational waves unless an EOS satisfies some specific criterion [their Eq. (74)]. We checked that this criterion is not equivalent and does not follow from our criterion $s=0$, which is a necessary condition for decoupling of superfluid modes from metric.

Conclusion. - Summarizing, equations describing pulsations of superfluid NSs can be split into two systems of weakly coupled equations. The coupling parameter $s$ of these systems is small for realistic EOSs, $|s| \sim 0.01-0.05$. One system of equations describes normal modes, another one - superfluid modes. Already zero approximation in parameter $s$ (when the systems are fully decoupled) is sufficient to calculate the pulsation spectrum with an accuracy of a few percent. In this 
approximation the normal modes coincide with ordinary modes of nonsuperfluid NS, while superfluid modes do not perturb metric, pressure, baryon current density and are localized in superfluid region of a star. Note that an emission of gravitational waves by superfluid modes is possible only in the next (first) order of perturbation theory in $s$. Thus, it should be suppressed in comparison to gravitational radiation from the normal modes.

Our finding that superfluid modes do not appear at the NS surface and do not emit gravitational waves in the $s=0$ limit indicate that these modes should be very difficult to observe at small but finite $s$. This means that observational properties of a pulsating superfluid star and a normal star of the same mass should be very similar, so that it will be very hard to discriminate one from the other.

The obtained results explain numerical calculations
[2, 3] and suggest simple perturbative (in parameter $s$ ) scheme which drastically simplifies the problem of calculation of the pulsation spectrum for superfluid NSs. The presented approach allows to easily take into account realistic EOSs, dissipation, various composition of matter, temperature effects, baryon superfluidity, densitydependent profiles of critical temperatures, and rotation of NSs. In more detail these issues will be discussed elsewhere [6].

Acknowledgements. - We thank D.P. Barsukov, A.I. Chugunov, and D.G. Yakovlev for valuable comments. This research was supported by the Dynasty Foundation, Ministry of Education and Science of Russian Federation (Contract No. 11.G34.31.0001 with SPbSPU and leading scientist G.G. Pavlov), RFBR (Grant No. 11-02-00253-a), and by FASI (Grant No. NSh$3769.2010 .2)$.
[1] N. Andersson, V. Ferrari, D. I. Jones et al., arXiv:0912.0384.

[2] L. Lindblom and G. Mendell, Astrophys. J. 421, 689 (1994).

[3] U. Lee, Astron. Astrophys. 303, 515 (1995); L. Lindblom and G. Mendell, Phys. Rev. D61, 104003 (2000); S. Yoshida and U. Lee, Phys. Rev. D67, 124019 (2003); L.-M. Lin, N. Andersson, and G. L. Comer, Phys. Rev. D78, 083008 (2008).

[4] N. Andersson, G. L. Comer, and D. Langlois, Phys. Rev. D66, 104002 (2002);

[5] See, however, Refs. [20, 21], where in two special cases and under a number of simplified assumptions the authors exactly decouple, in the nonrelativistic limit, superfluid degrees of freedom from the normal ones.

[6] M. E. Gusakov and E. M. Kantor, in preparation.

[7] I. M. Khalatnikov, An Introduction to the Theory of Superfluidity (Addison-Wesley, New York, 1989).

[8] M. E. Gusakov and N. Andersson, Mon. Not. R. Astron. Soc. 372, 1776 (2006).

[9] M. E. Gusakov, Phys. Rev. D76, 083001 (2007).

[10] M. E. Gusakov, E. M. Kantor, and P. Haensel, Phys. Rev. C79, 055806 (2009); Phys. Rev. C80, 015803 (2009).

[11] M. Prakash, J. M. Lattimer, and T. L. Ainsworth, Phys. Rev. Lett. 61, 2518 (1988).

[12] D. Page and J. H. Applegate, Astrophys. J. 394, L17
(1992)

[13] A. Akmal, V. R. Pandharipande, and D. G. Ravenhall, Phys. Rev. C58, 1804 (1998).

[14] E. Chabanat, P. Bonche, P. Haensel, J. Meyer, and R. Schaeffer, Nucl. Phys. A635, 231 (1998).

[15] L. G. Cao, U. Lombardo, C. W. Shen, and N. V. Giai, Phys. Rev. C73, 014313 (2006).

[16] J. M. Lattimer and M. Prakash, Phys. Rep. 442, 109 (2007).

[17] P. B. Demorest, T. Pennucci, S. M. Ransom, M. S. E. Roberts, and J. W. T. Hessels, Nature, 467, 1081 (2010).

[18] The maximum value $|s| \sim 0.1$ is reached for EOS PAL II180 at $\rho \sim 10^{15} \mathrm{~g} \mathrm{~cm}^{-3}$ (this region is not shown in Fig. 1). At such high densities hyperons are likely to appear in NS matter so that the simple semiphenomenological EOS which allows only for nucleons, is not applicable.

[19] I. L. Bekarevich and I. M. Khalatnikov, Zh. Eksp. Teor. Fiz. 39, 1699 (1960) [Sov. Phys. JETP 12, 1187 (1961)]; G. Mendell and L. Lindblom, Ann. Phys. 205, 110 (1991).

[20] R. Prix and M. Rieutord, Astron. Astrophys. 393, 949 (2002);

[21] B. Haskell, N. Andersson, and A. Passamonti, Mon. Not. R. Astron. Soc. 397, 1464 (2009). 